\title{
Transport of Heavy Quarks Across Glasma
}

\section{Stanisław Mrówczyński*}

Institute of Physics, Jan Kochanowski University, Kielce, Poland and National Centre for Nuclear Research, Warsaw, Poland

stanislaw.mrowczynskiencbj.gov.pl

The non-equilibrium quark-gluon system called 'glasma', which is dominated by classical chromodynamic fields, is produced at an earliest stage of ultrarelativistic heavy-ion collisions. We address the question of how heavy quarks interact with the glasma in comparison with the equilibrated quark-gluon plasma of the same energy density. For this purpose we derive a FokkerPlanck transport equation of heavy quarks embedded in QCD plasma. We first discuss the equilibrium plasma and then the glasma within the same approach where heavy quarks interact not with the plasma constituents but rather with long wavelength classical fields. We first consider the three schematic models of isotropic glasma and then the simplified model of glasma from ultrarelativistic heavy-ion collisions, where the chromodynamic fields are only along the beam direction. The momentum broadening and collisional energy loss of a test heavy quark are computed and compared to those of the equilibrium plasma of the same energy density.

International Conference on Hard and Electromagnetic Probes of High-Energy Nuclear Collisions 30 September - 5 October 2018

Aix-Les-Bains, Savoie, France

\footnotetext{
* Speaker.
} 
The early stage of relativistic heavy-ion collisions is least known because there are hardly any experimentally accessible signals of the phase. Nevertheless one expects that the system, which is produced in the collisions, is initially strongly populated with chromodynamic fields. Within the Color Glass Condensate (CGC) approach, see e.g. the review [1], the system is called 'glasma'. It occurs due to color charges confined in the colliding nuclei which act as sources of long wavelength chromodynamic fields. The fields can be treated classically because of their large occupation numbers. Since the density of color charges per transverse area of heavy nuclei is large, the corresponding momentum (saturation) scale $Q_{s}$ is expected to be significantly bigger than the QCD scale parameter $\Lambda_{\mathrm{QCD}}$. Consequently, the coupling constant $\alpha_{s}$ is presumably sufficiently small to allow for perturbative methods. The system, however, is rather strongly interacting because of the high-amplitude fields that prevail.

A momentum anisotropy of the early stage of quark-gluon plasma makes it unstable with respect to chromomagnetic modes which in turn cause a spontaneous generation of the fields, as explained at length in the review article [2]. Therefore, the effect of strong fields is further enhanced. Leaving aside the mechanism of field generation and its detailed structure, one asks what are transport properties of the glasma. We are specifically interested how heavy quarks - charm or beauty - behave in such a system when compared to the equilibrium plasma of the same energy density.

Heavy quarks are often treated as a probe of strongly interacting matter created in relativistic heavy-ion collisions, see e.g. the review [3]. Thanks to their large masses the quarks are produced only at the earliest stage of the collision due to hard interactions of partons from incoming nuclei. Later on they propagate through a surrounding medium testing the entire history of the system. It has been long believed that the interaction of heavy quarks in the medium is significantly weaker than that of light quarks or gluons but experimental data clearly contradict the expectation. As discussed in the review [3], the behavior of mesons containing a heavy quark is rather similar to that of light mesons at both small and large transverse momenta. The problem is not fully resolved.

The medium created in relativistic heavy-ion collisions evolves fast towards the locally equilibrated quark-gluon plasma which expands hydrodynamically and ultimately is converted into a hadron gas. Momentum spectra of heavy quarks from the final states are mostly shaped in the longlasting equilibrium phase which is relatively well understood. An effect of a pre-equilibrium phase is usually entirely ignored but this transient state can significantly influence heavy-quark spectra because of its high density. Non-equilibrium calculations recently performed in a framework of kinetic theory [4] confirm the suggestion. However, we are interested in even the earlier phase when the medium is not described in terms of quasi-particles, as in a kinetic theory, but the system is dominated by classical fields.

A simple parametric estimate suggests that the interaction of heavy quarks in the glasma is much stronger than in the equilibrium one, if the coupling constant $g$ is small. The momentum broadening parameter $\hat{q}$, for example, is of order $g^{4}$ in equilibrium plasmas. Since the quark of interest actually interacts with soft gluons emitted by plasma constituents, one can think that the factor $g^{4}$ is composed of two pieces of $g^{2}$. The first one is related to the gluon emission and the second one to the gluon absorption. If soft chromodynamic fields are present in the system, the interaction of the quark should be rather of order $g^{2}$ than $g^{4}$. We argue in [10] that $\hat{q}$ is indeed not of the order $g^{4}$, not even $g^{2}$ but it might be of the order $g$ in the glasma. The numerical effect, 
however, is not that impressive at a realistic value of the coupling constant.

Because of their big masses, relaxation times of heavy quarks, which are produced in relativistic heavy-ion collisions, are expected to be significantly longer than that of light quarks and gluons. When an equilibrium or, more generally, a stationary state is reached by light quarks and gluons, heavy quarks need some extra time to adjust to the state of the plasma. Such a situation is naturally described in terms of the Fokker-Planck transport equation which was indeed repeatedly applied to heavy quarks $[5,6,7,8]$. The equation is usually derived from the Boltzmann equation by applying the so-called diffusion approximation to the collision term [9]. The approximation assumes that the momentum transfer to a heavy quark in every collision is much smaller than the quark momentum.

The aim of our study [10], which is briefly presented here, was threefold. We firstly derived the Fokker-Planck equation of heavy quarks which do not interact with plasma constituents but rather with soft classical fields present in the plasma. Specifically, we applied the so-called quasi-linear theory known from the electromagnetic plasma $[9,11]$. The theory assumes that the distribution function can be decomposed into a large but slowly varying regular part and a small fluctuating or turbulent one which oscillates fast. The average over a statistical ensemble of the turbulent part is assumed to vanish and thus the average of the distribution function equals its regular part. The turbulent contribution to the distribution function obeys the collisionless transport equation while the transport equation of the regular part is determined by the fluctuation spectra which provide the collision term. Our derivation closely followed the procedure which had been developed for QCD in [12], where, however, only the longitudinal chromoelectric field had been taken into account. In the study [10] the complete chromodynamic field was considered. The equilibrium correlation functions of chromodynamic fields, which were needed to obtain the quasi-linear transport equations, had been derived in [13]. In case of equilibrium plasma we reproduced the Fokker-Planck equation derived long ago in [6] from the Boltzmann equation by means of the diffusive approximation. The result is far not trivial as our dynamical input was very different than that in [6] where matrix elements of parton binary processes were used.

Our second aim was to compare glasma transport coefficients to those of equilibrium plasma at the same energy density. For this purpose we considered three models of isotropic glasma postulating a form of the correlation functions of chromodynamic fields. We derived the coefficients of the Fokker-Planck equation which are related to the energy loss $d E / d x$, momentum broadening $\hat{q}$ and diffusion coefficient $D$ of heavy quarks. Although the values of $d E / d x$ and $\hat{q}$ strongly depend on how the glasma is modeled, the transport coefficients are significantly bigger than their equilibrium counterparts.

The third aim of our study [10] was to discuss an evolution of heavy quarks at the earliest stage of relativistic heavy-ion collisions. Since the chromoelectric and chromomagnetic fields spanned between the receding nuclei are initially mostly parallel to the beam direction, we modeled the glasma with the boost invariant correlation functions of the longitudinal fields. The energy loss and momentum broadening of heavy quarks were computed under the assumption that the whole energy of colliding nuclei, which is ultimately converted into energy of produced particles, is initially accumulated in the longitudinal fields present in the glasma. We obtained the following estimates

$$
-\frac{d E}{d x} \approx 14 v \cos ^{2} \theta\left[\frac{\mathrm{GeV}}{\mathrm{fm}}\right], \quad \hat{q} \approx 33 \frac{\sin ^{2} \theta+v \sin \theta}{v}\left[\frac{\mathrm{GeV}^{2}}{\mathrm{fm}}\right]
$$


where $v$ is the heavy quark velocity and $\theta$ is the angle between the velocity and the beam direction. Since in our simplistic model the chromoelectric and chromomagnetic fields are only along the beam axis, there is a peculiar directional dependence of $d E / d x$ and $\hat{q}$. The energy loss is maximal when the test heavy quark moves along the beam $(\theta=0)$ and the momentum broadening is maximal when the quark moves perpendicularly to the beam $(\theta=\pi / 2)$.

The numbers (1) should be compared to those which are required to properly model experimental data on the charm meson suppression. The collisional energy loss and momentum broadening of a charm quark with $10 \mathrm{GeV}$ momentum in the plasma of the temperature from the interval $0.35-0.5 \mathrm{GeV}$ are estimated [3] as

$$
-\frac{d E}{d x}=1.0-3.0\left[\frac{\mathrm{GeV}}{\mathrm{fm}}\right], \quad \hat{q}=1.5-7.0\left[\frac{\mathrm{GeV}^{2}}{\mathrm{fm}}\right] .
$$

As seen, the values (1) can be significantly larger than (2), suggesting that in spite of a short lifetime of the glasma it can provide a significant contribution to the collisional and radiative energy loss of heavy quarks produced in relativistic heavy-ion collisions. Consequently, the effect should be included in the phenomenology of jet quenching.

The findings presented in our study [10] clearly suggest a direction of further work. We need a more realistic model of glasma where a temporal evolution of the system is taken into account and the fields are not purely longitudinal. The CGC studies reviewed in [1] and, in particular, the analytic analysis [14] provide a very good guidance for such a model which is now under construction.

\section{References}

[1] F. Gelis, Int. J. Mod. Phys. A 28, 1330001 (2013).

[2] St. Mrówczyński, B. Schenke and M. Strickland, Phys. Rept. 682, 1 (2017).

[3] F. Prino and R. Rapp, J. Phys. G 43, 093002 (2016).

[4] S. K. Das, M. Ruggieri, F. Scardina, S. Plumari and V. Greco, J. Phys. G 44, 095102 (2017).

[5] G. D. Moore and D. Teaney, Phys. Rev. C 71, 064904 (2005).

[6] B. Svetitsky, Phys. Rev. D 37, 2484 (1988).

[7] H. van Hees and R. Rapp, Phys. Rev. C 71, 034907 (2005).

[8] M. G. Mustafa, Phys. Rev. C 72, 014905 (2005).

[9] E. M. Lifshitz and L. P. Pitaevskii, Physical Kinetics (Pergamon Press, Oxford, 1981).

[10] St. Mrówczyński, Eur. Phys. J. A 54, 43 (2018).

[11] A. A. Vedenov, E. P. Velikhov and R. Z. Sagdeev, Usp. Fiz. Nauk, 73, 701 (1961) [in Russian]; Sov. Phys. Usp. 4, 332 (1961).

[12] St. Mrówczyński and B. Müller, Phys. Rev. D 81, 065021 (2010).

[13] St. Mrówczyński, Phys. Rev. D 77, 105022 (2008).

[14] G. Chen, R. J. Fries, J. I. Kapusta and Y. Li, Phys. Rev. C 92, 064912 (2015). 